\title{
SELF-GRAVITATING GAS DYNAMICS \\ IN A NUCLEAR REGION OF A WEAK BARRED GALAXY
}

\author{
K. WADA A. HABE \\ Department of Physics, Hokkaido University, Sappro 060 Japan
}

\section{GAS FUELING MECHANISM}

Starburst regions are frequently located in galactic central regions and $\mathrm{CO}$ observations indicate that these regions contain a large amount of molecular gas(e.g. Lo et al. 1987). However, the triggering mechanism for starbursts and the mechanism of the high mass supply rate of gas into a galactic center are still unclear.

It is suggested that tidal encounters of galaxies remove angular momentum of gas and trigger rapid gas accretion and starburst. Noguchi(1988) has shown by computer simulations that galaxy-galaxy interactions induce a stellar bar, and gas loses its angular momentum and accumulates to a galactic center. In his numerical simulation, non-axisymmetric potential of a stellar bar plays an important role in the accretion of gas. However, it is not obvious whether or not gas accretes into a nuclear region within a few hundred pc only by the effects of stellar bar.

Wada and Habe(1992) investigated the dynamics of self-gravitating gas in a barred potential by 2-D numerical simulation, and show that even if the bar is weak, for the initial gas mass ratio to stellar mass grater than $10 \%$, a central elongated gas ring formed at near ILRs becomes unstable and collapses. As a result, a large amount of gas can be supplied to galactic center. They conclude that both the effect of the self-gravity of the gas and the existence of ILRs are necessary to the rapid gas fueling in a weak barred potential.

\section{GAS DYNAMICS IN THE STARBURST REGION}

To analyze this rapid and complex process of the gas fueling, we made a video of above simulation. The video clearly shows that the formation of dense gas clumps at both sides of the elongated ring triggers instability of the ring: The dense clumps move and collide with each other on a highly elongated orbit; After frequent collisions, these gas clumps merge and finally form a central dense core.

Recent CO observations with high spacial resolution reveal that the structure and kinematics of molecular gas in the starburst galaxies(Ishizuki 1992, and see papers by Levine et al. , Handa et al. , Turner, and Kenney in this proceedings). The molecular gas has a large non-circular motion in many starburst galaxies, and also has various kinds of structure such as a bar/ridge( NGC2782, IC342 and M83), double peaks (NGC3504, 2782, 6951, 3351, and M101), and a ring( $M 82$ and NGC1068). Is there a unified theory to explain all these feature? 
In our simulation we find that the high density region of the dense core of gas changes its structure: an elongated ring or bar-like feature appears by turns in a very short timescale $\left(\sim 10^{6-7} \mathrm{yr}\right)$. The observed feature in starburst galaxies can be understood as the time sequence of the non-axisymetric kinematics in our model.

On the other hand, there is an argument to explain the dynamics of the molecular gas by the theory of the closed orbit families $(x 1$ and $x 2$ in the notation of Contopulos), as the study about our Galaxy by Binney et al. (1991). However, we should be careful to apply this argument to the molecular gas in the central region of the starburst galaxies. Because the argument based on the liner theory probably breaks down in the region in which the self-gravity of the gas cannot be neglected for the gas dynamics. To get the complete understanding of the gas dynamics in the central region of the starburst galaxies, we are preparing for self-consistent studies taking into account both gas and stellar component not only by 2-D but also by $3-\mathrm{D}$ simulations.

\section{REFERENCES}

Binney, J. et al. 1991. Mon. Not. R. astr. Soc., 252, 210.

Ishizuki, S. et al. 1990. Nature, 344, 224.

Lo, K.Y. et al. 1987. Astrophys. J., 312, 574.

Noguchi, M.,1988. Astr. Astrophys., 203, 259.

Wada, K. \& Habe, A.,1992. Mon. Not. R. astr. Soc., 258, 82.
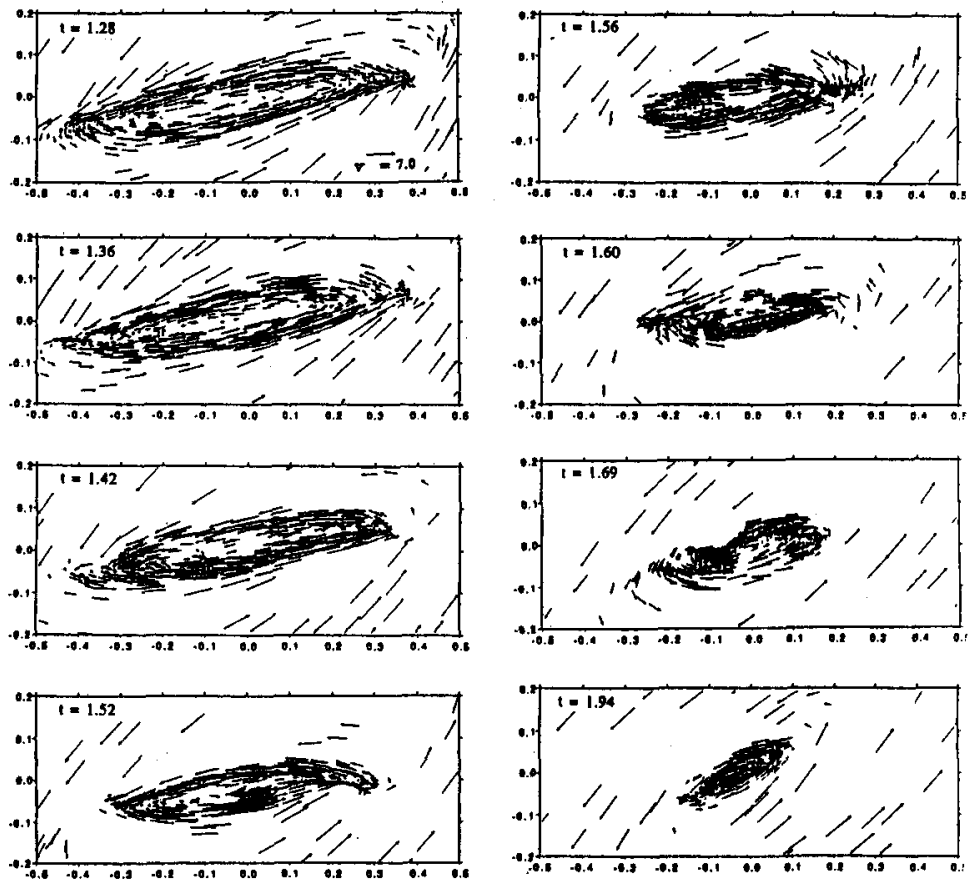

FIGURE I Collapse of the gas ring. The arrows represent the velocity vector of the gas. The unit of the time is $3.5 \times 10^{8} \mathrm{yr}$. 